Bull. Austral. Math. Soc.

$46 \mathrm{~L} 05,46 \mathrm{~L} 55$

Vol. 75 (2007) [229-238]

\title{
EXTENSION PROBLEMS AND NON-ABELIAN DUALITY FOR $C^{*}$-ALGEBRAS
}

\author{
Astrid an Huef, S. Kaliszewski and Iain Raeburn
}

Suppose that $H$ is a closed subgroup of a locally compact group $G$. We show that a unitary representation $U$ of $H$ is the restriction of a unitary representation of $G$ if and only if a dual representation $\widehat{U}$ of a crossed product $C^{*}(G) \rtimes(G / H)$ is regular in an appropriate sense. We then discuss the problem of deciding whether a given representation is regular; we believe that this problem will prove to be an interesting test question in non-Abelian duality for crossed products of $C^{*}$-algebras.

In [9], we considered the classical problem of deciding when a unitary representation $U$ of a closed normal subgroup $H$ of a locally compact group $G$ extends to a unitary representation of $G$ on the same space. We proved that $U$ extends to $G$ if and only if a dual representation $\widehat{U}$ of a coaction crossed product $C^{*}(G) \rtimes(G / H)$ is regular, and used Mansfield's imprimitivity theorem [12] to describe these regular representations when $G$ is amenable [9, Theorem 5]. Since regular representations are induced representations, this accords with a general principle in non-Abelian duality for crossed products of $C^{*}$ algebras: duality swaps restriction and induction of representations $[2,11,4]$. The extension problem makes sense for an arbitrary (not necessarily normal) closed subgroup $H$, and finding the appropriate dual formulation is an intriguing test question for any theory of crossed products by homogeneous spaces.

In general we do not know what it means to say that a homogeneous space $G / H$ coacts on a $C^{*}$-algebra, but when we have a coaction of $G$ on a $C^{*}$-algebra $A$, we can define a crossed product $C^{*}$-algebra $A \rtimes(G / H)$, and such algebras arise in applications (see, for example, $[6,1,13])$. The coaction arising in the above extension problem is the restriction of a dual coaction of $G$, and the crossed product $C^{*}(G) \rtimes(G / H)$ has a natural family of regular representations. Our Theorem 1 says that $U$ extends to a unitary representation of $G$ if and only if $\widehat{U}$ is regular in this sense. The coactions are only needed to motivate the result, which can be formulated using only ordinary crossed products by actions, and its proof uses a direct spatial argument.

Received 16th October, 2006

This research was supported by grants from the Australian Research Council, the National Science Foundation and the University of New South Wales.

Copyright Clearance Centre, Inc. Serial-fee code: 0004-9727/07 \$A2.00+0.00. 
Theorem 1 raises the question of how one can decide whether a given representation of a crossed product $C^{*}(G) \rtimes(G / H)$ is regular. Our Theorem 2 answers this question when $H$ is normal, and gives a version of [9, Theorem 5] which works for non-amenable groups (see Corollary 4). We have also been able to make some progress on this question in the purely algebraic situation where $H$ is not normal but $(G, H)$ is a discrete Hecke pair; since this has required completely different methods, we discuss these results elsewhere [8].

Our results sit naturally in the context of $C^{*}$-dynamical systems consisting of an action $\alpha$ of a locally compact group $G$ by automorphisms of a $C^{*}$-algebra $A$, and we work in this generality throughout.

\section{Preliminaries}

Throughout, $H$ is a closed subgroup of a locally compact group $G$, and $N$ is a closed normal subgroup. The group $G$ acts on the left of the homogeneous space $G / H$, and this induces a continuous action $\mathrm{lt}: G \rightarrow$ Aut $C_{0}(G / H)$ defined by $\operatorname{lt}_{t}(f)(s H)=f\left(t^{-1} s H\right)$. A representation $V$ of $G$ is a continuous homomorphism $V$ of $G$ into the group $U(\mathcal{H})$ of unitary operators on a Hilbert space $\mathcal{H}$, where $U(\mathcal{H})$ has the strong operator topology.

We use left Haar measures, and denote by $\Delta_{G}$ and $\Delta_{H}$ the modular functions on $G$ and $H$. Let $\rho: G \rightarrow(0, \infty)$ be a continuous function such that

$$
\rho(s t)=\frac{\Delta_{H}(t)}{\Delta_{G}(t)} \rho(s)
$$

for $s \in G$ and $t \in H$. From [14, Section C.1], for example, there exists a quasi-invariant measure on the quotient space $G / H$ such that

$$
\int_{G} f(s) \rho(s) d s=\int_{G / H} \int_{H} f(s t) d t d(s H) \text { for } f \in C_{c}(G) .
$$

If $N$ is a closed normal subgroup of $G$ with $N \subset H$ then $\Delta_{H}(n)=\Delta_{N}(n)=\Delta_{G}(n)$ and hence $\rho(s n)=\rho(s)$ for all $n \in N$ and $s \in G$. We can thus choose left Haar measures on $G / N$ and $H / N$ such that

$$
\int_{G / N} f(s N) \rho(s) d(s N)=\int_{G / H} \int_{H / N} f(s t N) d(t N) d(s H) \text { for } f \in C_{c}(G / N) .
$$

We denote by $\lambda^{G / H}$ the quasi-regular representation of $G$ on $L^{2}(G / H)$ given by

$$
\left(\lambda_{r}^{G / H} \xi\right)(s H)=\sqrt{\frac{\rho\left(r^{-1} s\right)}{\rho(s)}} \xi\left(r^{-1} s H\right) \quad \text { for } s, r \in G \text { and } \xi \in L^{2}(G / H),
$$

and we denote by $M$ the representation of $C_{0}(G / H)$ by multiplication operators on $L^{2}(G / H)$. We use $\lambda$ and $\nu$ to denote left- and right-regular representations, respectively; 
so, for example, $\lambda$ could denote the left regular representation of $G$ on $L^{2}(G)$, or of $G / N$ on $L^{2}(G / N)$.

Our conventions regarding actions and coactions of $G$ on a $C^{*}$-algebra $A$ are those of $[4$, Appendix A]. We emphasise that all the group algebras and crossed products in this paper are full; in other words, they are universal for appropriate families of covariant representations.

We denote by $X$ or $X_{H}^{G}(A)$ the $\left(\left(A \otimes C_{0}(G / H)\right) \rtimes_{\alpha \otimes \mathfrak{l t}} G\right)-\left(A \rtimes_{\alpha} H\right)$ imprimitivity bimodule implementing Green's imprimitivity theorem [7, Theorem 6]. We view $X$ as the completion of $X_{0}:=C_{c}(G, A)$ with respect to the formulas for the actions and inner products from [4, Equations (B.5)]. Tensoring with $X$ then gives a bijection

$$
X \text {-Ind }: \operatorname{Rep}\left(A \rtimes_{\alpha} H\right) \rightarrow \operatorname{Rep}\left(\left(A \otimes C_{0}(G / H)\right) \rtimes_{\alpha \otimes \mid \mathrm{t}} G\right)
$$

on unitary equivalence classes of representations [14, Theorem 3.29].

\section{CoACTION-REgUlar REPRESENTATIONS AND THE EXTENSION PROBLEM}

Suppose $(A, G, \alpha)$ is a dynamical system, $H$ is a closed subgroup of $G$, and $(\pi, U)$ is a covariant representation of $(A, H, \alpha)$. We want to find a condition on the induced representation $X$-Ind $(\pi \rtimes U)$ of $\left(A \otimes C_{0}(G / H)\right) x_{\alpha \otimes \mid \mathrm{t}} G$ which is equivalent to saying that $(\pi, U)$ extends to a covariant representation of $(A, G, \alpha)$. Our result will say that $(\pi, U)$ extends if and only if $X$-Ind $(\pi \rtimes U)$ is a kind of regular representation. However, to see why we call these representations regular we have to view $\left(A \otimes C_{0}(G / H)\right) \rtimes_{\alpha \otimes l t} G$ as a crossed product of $A \rtimes_{\alpha} G$ by a coaction of the homogeneous space $G / H$. As motivation for this, we look at what happens when we have a closed normal subgroup $N$.

Denote by $\hat{\alpha}: A \rightarrow M\left(\left(A \rtimes_{\alpha} G\right) \otimes C^{*}(G)\right)$ the dual coaction of $G$ on the crossed product $A \rtimes_{\alpha} G[4, A .26]$. Since the subgroup $N$ is normal, we can restrict $\widehat{\alpha}$ to a coaction $\widehat{\alpha} \mid$ of $G / N$ on $A \rtimes_{\alpha} G$ [4, A.28]. It is proved in [4, Proposition A.63 and Theorem A.64], for example, that there is an isomorphism of $\left(A \rtimes_{\alpha} G\right) \rtimes_{\widehat{\alpha} \mid} G / N$ onto $\left(A \otimes C_{0}(G / N)\right) \rtimes_{\alpha \otimes \mid \mathrm{t}} G$ which carries a representation $(\phi \rtimes W) \rtimes \mu$ of $\left(A \rtimes_{\alpha} G\right) \rtimes_{\widehat{\alpha} \mid} G / N$ into the representation $(\phi \otimes \mu) \rtimes W$ of $\left(A \otimes C_{0}(G / N)\right) \rtimes_{\alpha \otimes \mid t} G$. The regular representation of $\left(A \rtimes_{\alpha} G\right) \rtimes_{\widehat{\alpha} \mid} G / N$ induced from a covariant representation $(\phi, W)$ of $(A, G, \alpha)$ is the representation

$$
(((\phi \rtimes W) \otimes \lambda) \circ \widehat{\alpha} \mid) \rtimes(1 \otimes M)
$$

on $\mathcal{H} \otimes L^{2}(G / N)$, and we call the corresponding representation

$$
(\phi \otimes M) \rtimes\left(W \otimes \lambda^{G / N}\right)
$$

of $\left(A \otimes C_{0}(G / N)\right) \rtimes_{\alpha \otimes 1 \mathrm{t}} G$ the coaction-regular representation induced from $(\phi, W)$.

When $H$ is not normal, we do not have a satisfactory notion of coaction of $G / H$, and hence no completely satisfactory notion of crossed product by $G / H$. However, here we 
have a coaction $\hat{\alpha}$ of $G$, and $\left(A \otimes C_{0}(G / H)\right) \rtimes_{\alpha \otimes 1 t} G$ is one of the candidates for what we should mean by the crossed product of $A \rtimes_{\alpha} G$ by the coaction $\widehat{\alpha}$ of the homogeneous space $G / H$ (see the discussion in [5, Section 2]). Further, we can still use the quasi-regular representation $\lambda^{G / H}$ of $G$ to build a representation

$$
(\phi \otimes M) \rtimes\left(W \otimes \lambda^{G / H}\right):\left(A \otimes C_{0}(G / H)\right) \rtimes_{\alpha \otimes \mid \mathrm{t}} G \rightarrow B\left(\mathcal{H} \otimes L^{2}(G / H)\right),
$$

and, as in the normal case, we call this the coaction-regular representation induced from $(\phi, W)$. We do this to emphasise that it is only regular when we think of $\left(A \otimes C_{0}(G / H)\right) \rtimes_{\alpha \otimes l \mathrm{t}} G$ as a crossed product by $G / H$; the regular representations of $\left(A \otimes C_{0}(G / H)\right) \rtimes_{\alpha \otimes \mid t} G$ induced from representations of $A \otimes C_{0}(G / H)$ are quite different, and it is the coaction-regular representations which arise naturally in our dual problem.

THEOREM 1. Suppose that $\alpha$ is an action of a locally compact group $G$ on a $C^{*}$-algebra $A$ and $H$ is a closed subgroup of $G$. Let $X$ be the imprimitivity bimodule implementing Green's imprimitivity theorem, and let $(\pi, U)$ be a covariant representation of $(A, H, \alpha)$ on a Hilbert space $\mathcal{H}$. Then there exists a covariant representation $(\pi, V)$ of $(A, G, \alpha)$ on $\mathcal{H}$ such that $U=\left.V\right|_{H}$ if and only if $X-\operatorname{Ind}(\pi \rtimes U)$ is unitarily equivalent to a coaction-regular representation.

Proof: We shall show that for any covariant representation $(\pi, V)$ of $(A, G, \alpha)$, we have

$$
X-\operatorname{Ind}\left(\left.\pi \rtimes V\right|_{H}\right) \sim(\pi \otimes M) \rtimes\left(V \otimes \lambda^{G / H}\right) .
$$

This immediately gives the "only if" direction of the theorem. It also gives the converse: if $X$-Ind $(\pi \rtimes U)$ is unitarily equivalent to the coaction-regular representation induced from some covariant representation $(\phi, W)$, then (2.1) gives

$$
X-\operatorname{Ind}\left(\left.\phi \rtimes W\right|_{H}\right) \sim(\phi \otimes M) \rtimes\left(W \otimes \lambda^{G / H}\right) \sim X-\operatorname{Ind}(\pi \rtimes U),
$$

so that $\pi \rtimes U$ is unitarily equivalent to $\left.\phi \rtimes W\right|_{H}$ because $X$ is an imprimitivity bimodule. Now moving $W$ over to the space of $(\pi, U)$ gives the desired representation $V$.

So suppose that $(\pi, V)$ is a representation of $(A, G, \alpha)$ and $U=\left.V\right|_{H}$. We define $\Psi: X_{0} \odot_{A \rtimes_{\alpha} H} \mathcal{H} \rightarrow L^{2}(G / H, \mathcal{H})$ by

$$
\Psi(x \otimes h)(s H)=\int_{H} \pi(x(s t)) V_{s t} h \Delta_{G}(s t)^{-1 / 2} \rho(s t)^{-1 / 2} d t ;
$$

the usual arguments show that $\Psi(x \otimes h)$ is a continuous function of compact support, and hence belongs to $L^{2}(G / H, \mathcal{H})$. We claim that $\Psi$ extends to a unitary isomorphism of $X \otimes_{A \rtimes_{\alpha} H} \mathcal{H}$ onto $L^{2}(G / H, \mathcal{H})$ which intertwines $X$-Ind $(\pi \rtimes U)$ and $(\pi \otimes M) \rtimes\left(V \otimes \lambda^{G / H}\right)$. 
We start by checking that $\Psi$ is isometric. Let $x, y \in X_{0}$ and $h, k \in \mathcal{H}$. Then using the formulas for the inner product in $[4,(B .5)]$, we have

$$
\begin{aligned}
(x \otimes h \mid y \otimes k) & =\left(\pi \rtimes U\left(\langle y, x\rangle_{A \rtimes_{\alpha} H}\right) h \mid k\right) \\
& =\left(\left(\int_{H} \pi\left(\langle y, x\rangle_{A \rtimes_{\alpha} H}(t)\right) U_{t} d t\right) h \mid k\right) \\
& =\int_{H} \int_{G}\left(\pi\left(\alpha_{s}\left(y\left(s^{-1}\right)^{*} x\left(s^{-1} t\right)\right)\right) U_{t} h \mid k\right) \Delta_{H}(t)^{-1 / 2} d s d t \\
& =\int_{H} \int_{G}\left(\pi\left(\alpha_{s}\left(x\left(s^{-1} t\right)\right)\right) U_{t} h \mid \pi\left(\alpha_{s}\left(y\left(s^{-1}\right)\right)\right) k\right) \Delta_{H}(t)^{-1 / 2} d s d t \\
& =\int_{H} \int_{G}\left(\pi\left(x\left(s^{-1} t\right)\right) V_{s-1_{t}} h \mid \pi\left(y\left(s^{-1}\right)\right) V_{s-1} k\right) \Delta_{H}(t)^{-1 / 2} d s d t
\end{aligned}
$$

using the covariance of $(\pi, V)$ for $(A, G, \alpha)$ and $U_{t}=V_{t}$ for $t \in H$. At the expense of a modular function we replace $s$ by $s^{-1}$, and then we write the integral over $G$ as an iterated integral over $G / H$ and $H$. This gives

$$
\begin{aligned}
(2.3) & =\int_{H} \int_{G / H} \int_{H}\left(\pi(x(s r t)) V_{s r t} h \mid \pi(y(s r)) V_{s r} k\right) \Delta_{G}(s r)^{-1} \Delta_{H}(t)^{-1 / 2} \rho(s r)^{-1} \\
& =\int_{H} \int_{G / H} \int_{H}\left(\pi(x(s t)) V_{s t} h \mid \pi(y(s r)) V_{s r} k\right) \Delta_{G}(s r)^{-1} \Delta_{H}\left(r^{-1} t\right)^{-1 / 2} \rho(s r)^{-1} \\
& d r d(s H) d t .
\end{aligned}
$$

Since $\rho(s t) \Delta_{G}(t)=\rho(s) \Delta_{H}(t)$ for $s \in G$ and $t \in H$, we have

$$
\Delta_{G}(s r)^{-1} \Delta_{H}\left(r^{-1} t\right)^{-1 / 2} \rho(s r)^{-1}=\Delta_{G}(s r)^{-1 / 2} \rho(s r)^{-1 / 2} \Delta_{G}(s t)^{-1 / 2} \rho(s t)^{-1 / 2},
$$

and an application of Fubini's Theorem gives

$$
\begin{aligned}
(2.4)=\int_{G / H}\left(\int_{H} \pi(x(s t)) V_{s t} h \rho(s t)^{-1 / 2} \Delta_{G}(s t)^{-1 / 2} d t\right. \\
\\
\left.\mid \int_{H} \pi(y(s r)) V_{s r} k \rho(s r)^{-1 / 2} \Delta_{G}(s r)^{-1 / 2} d r\right) d(s H),
\end{aligned}
$$

which is $(\Psi(x \otimes h) \mid \Psi(y \otimes k))$. Thus $\Psi$ is isometric, and extends to an isometric linear map of $X \otimes_{A \rtimes_{\alpha} H} \mathcal{H}$ into $L^{2}(G / H, \mathcal{H})$.

To see that $\Psi$ has dense range, let $\xi \in C_{c}(G / H)$ be nonzero, and fix $h \in \mathcal{H}$; since $C_{c}(G / H) \odot \mathcal{H}$ is dense in $L^{2}(G / H) \otimes \mathcal{H} \cong L^{2}(G / H, \mathcal{H})$, it suffices to approximate $\xi \otimes h: s H \mapsto \xi(s H) h$ in the inductive limit topology by functions of the form $\sum_{i=1}^{n} \Psi\left(x_{i} \otimes h_{i}\right)$ for $x_{i} \in X_{0}$ and $h_{i} \in \mathcal{H}$. There exists nonzero $f \in C_{c}(G)$ such that (supp $\left.f\right) / H=\operatorname{supp} \xi$ and

$$
\xi(s H)=I(f)(s H):=\int_{H} f(s t) d t .
$$


Fix $\varepsilon>0$ and a relatively compact open neighbourhood $K$ of $\operatorname{supp} f$. Since $\pi$ is nondegenerate, an approximate identity argument shows that there exists $a \in A$ such that $\|\pi(a) h-h\|<\varepsilon / 3$, and now an $\varepsilon / 3$ argument implies that there exists a neighbourhood $O$ of the identity in $G$ such that

$$
s \in O \Longrightarrow\left\|\pi(a) V_{s} h-h\right\|<\varepsilon /\left(\|I(f)\|_{\infty} \mathrm{m}(\operatorname{supp} f)\right) .
$$

Choose $s_{i} \in G$ such that $\bigcup_{i=1}^{n} O s_{i}$ contains $K$, and use a partition of unity to find functions $\left\{p_{i}: 1 \leqslant i \leqslant n\right\}$ in $C_{c}(G)$ such that supp $p_{i} \subset O s_{i} \cap K$ and $\sum_{i} p_{i}=1$ on supp $f$. Then $\xi=I(f)=\sum_{i=1}^{n} I\left(p_{i} f\right)$. Let $x_{i}=p_{i} f \Delta_{G}^{1 / 2} \rho^{1 / 2} \otimes a$. Note that supp $x_{i} \subset K$, and hence the support of $\sum_{i=1}^{n} \Psi\left(x_{i} \otimes V_{s_{i}}^{*} h\right)$ is contained in $K / H$. For $s H \in K / H$ we have

$$
\begin{aligned}
\left\|\xi \otimes h(s H)-\sum_{i=1}^{n} \Psi\left(x_{i} \otimes V_{s_{i}}^{*} h\right)(s H)\right\| & \leqslant \sum_{i=1}^{n} \int_{H}\left|p_{i}(s t) f(s t)\right|\left\|h-\pi(a) V_{s t s_{i}^{-1}} h\right\| d t \\
& <\frac{\varepsilon}{\|I(f)\|_{\infty} \mathrm{m}(\operatorname{supp} f)} \sum_{i=1}^{n} \int_{H}\left|p_{i}(s t) f(s t)\right| d t \leqslant \varepsilon .
\end{aligned}
$$

because the first integrand vanishes unless $p_{i}(s t) \neq 0$, in which case $s t s_{i}^{-1} \in O$ and (2.5) applies. We have now proved that $\Psi$ extends to a unitary isomorphism of $X \otimes_{A \rtimes_{\alpha} H} \mathcal{H}$ onto $L^{2}(G / H, \mathcal{H})$.

To finish the proof we verify that $\Psi$ intertwines the two representations. Let $b$ belong to the dense subalgebra $C_{c}(G \times G / H, A)$ of $\left(A \otimes C_{0}(G / H)\right) \rtimes_{\alpha \otimes \mathrm{t}} G$. Then

$$
\begin{aligned}
(((\pi & \left.\left.\otimes M) \rtimes\left(V \otimes \lambda^{G / H}\right)\right)(b) \Psi(x \otimes h)\right)(s H) \\
& =\int_{G}(\pi \otimes M)(b(r, \cdot))\left(V_{r} \otimes \lambda_{r}^{G / H}\right)(\Psi(x \otimes h))(s H) d r \\
& =\int_{G} \pi(b(r, s H)) V_{r}\left(\int_{H} \pi\left(x\left(r^{-1} s t\right)\right) V_{r^{-1} s t} h \Delta_{G}\left(r^{-1} s t\right)^{-1 / 2} \rho\left(r^{-1} s t\right)^{-1 / 2} d t\right) \\
& \left.=\int_{G} \int_{H} \pi(b(r, s H)) V_{r} \pi\left(x\left(r^{-1} s t\right)\right) V_{r-1} s\right)^{1 / 2} \rho(s)^{-1 / 2} d r \\
& =\int_{G} \int_{H} \pi(b(r, s H)) \pi\left(r^{-1} s t\right)^{-1 / 2} \rho(s t)^{-1 / 2} d t d r \\
& =\int_{H} \pi\left(\int_{G} b(r, s H) \alpha_{r}\left(x\left(r^{-1} s t\right)\right)\right) V_{s t} h \Delta_{G}\left(r^{-1} s t\right)^{-1 / 2} \rho(s t)^{-1 / 2} d t d r
\end{aligned}
$$

by Fubini's Theorem. Upon recognising the inner integral as $b \cdot x(s t)$, where $b \cdot x$ denotes the left action of $b \in\left(A \otimes C_{0}(G / H)\right) \rtimes_{\alpha \otimes \mid \mathrm{t}} G$ on $x \in X$, this becomes

$$
\Psi((b \cdot x) \otimes h)(s H)=\Psi((X-\operatorname{Ind}(\pi \rtimes U)(b))(x \otimes h))(s H),
$$


as required.

From now on let $N$ be a closed normal subgroup $N$ of $G$. Our goal is to decide when a representation of $\left(A \otimes C_{0}(G / N)\right) \rtimes_{\alpha \otimes \text { it }} G$ is coaction-regular, and then to combine this with Theorem 1 to get a solution to - or at least a reformulation of - the original extension problem.

To state our next theorem, we need some notation. The action id $\otimes \mathrm{rt}$ of $G / N$ by right translation on $A \otimes C_{0}(G / N)$ commutes with $\alpha \otimes \mathrm{lt}$, and hence induces an action $\beta$ of $G / N$ on $\left(A \otimes C_{0}(G / N)\right) \rtimes_{\alpha \otimes 1 \mathrm{t}} G$ such that

$$
\beta_{t N}(b)(s, r N)=b(s, r t N) \text { for } b \in C_{c}(G \times G / N, A) .
$$

THEOREM 2. Suppose that $\alpha$ is an action of a locally compact group $G$ on a $C^{*}$ algebra $A, N$ is a closed normal subgroup of $G$, and $\pi:\left(A \otimes C_{0}(G / N)\right) \rtimes_{\alpha \otimes 1 \mathrm{t}} G \rightarrow B(\mathcal{H})$ is a representation. Then $\pi$ is unitarily equivalent to a coaction-regular representation if and only if there exists a representation $S$ of $G / N$ on $\mathcal{H}$ such that $(\pi, S)$ is a covariant representation of $\left(\left(A \otimes C_{0}(G / N)\right) \rtimes_{\alpha \otimes \mathrm{lt}} G, G / N, \beta\right)$.

Proof: If we start with a coaction-regular representation

$$
(\phi \otimes M) \rtimes\left(W \otimes \lambda^{G / N}\right):\left(A \otimes C_{0}(G / N)\right) \rtimes_{\alpha \otimes \mid \mathrm{t}} G \rightarrow B\left(\mathcal{H} \otimes L^{2}(G / N)\right),
$$

then the right-regular representation $S=1 \otimes \nu$ has the required property. So if $\pi$ is unitarily equivalent to $(\phi \otimes M) \rtimes\left(W \otimes \lambda^{G / N}\right)$, we just need to move $1 \otimes \nu$ to the space of $\pi$ to obtain $S$.

Conversely, suppose that $(\pi, S)$ is covariant for $\left(\left(A \otimes C_{0}(G / N)\right) \rtimes_{\alpha \otimes l \mathrm{t}} G, G / N, \beta\right)$, and decompose $\pi$ as $\left(\pi_{1} \otimes \mu\right) \rtimes U$. Notice that $S$ commutes with $U$ and gives a covariant representation $\left(\pi_{1} \otimes \mu, S\right)$ of $\left(A \otimes C_{0}(G / N), G / N\right.$, id $\left.\otimes \mathrm{rt}\right)$. We want to find a Hilbert space $\mathcal{H}_{2}$, an isomorphism $Y: \mathcal{H} \rightarrow \mathcal{H}_{2} \otimes L^{2}(G / N)$ and a covariant representation $\left(\pi_{2}, W\right)$ of $(A, G, \alpha)$ on $\mathcal{H}_{2}$ such that

$$
Y\left(\pi_{1} \otimes \mu, U, S\right) Y^{*}=\left(\pi_{2} \otimes M, W \otimes \lambda^{G / N}, 1 \otimes \nu\right) .
$$

(There is a notational subtlety here: the $\pi_{1} \otimes \mu$ on the left-hand side of (2.7) is the representation of $A \otimes C_{0}(G / N)=A \otimes_{\max } C_{0}(G / N)$ associated to the two commuting representations $\pi_{1}$ and $\mu$ on the same space, whereas the $\pi_{2} \otimes M$ on the right-hand side of (2.7) is the representation of $A \otimes C_{0}(G / N)=A \otimes_{\sigma} C_{0}(G / N)$ associated to the representations $\pi_{2}$ and $M$ on the different spaces $\mathcal{H}_{2}$ and $L^{2}(G / N)$.)

Applying the Stone-von Neumann theorem (as in [14, Remark C.35], for example) to the representation $(\mu, S)$ of $\left(C_{0}(G / N), G / N\right.$, rt $)$ gives a Hilbert space $\mathcal{H}_{2}$ and an isomorphism $Y: \mathcal{H} \rightarrow \mathcal{H}_{2} \otimes L^{2}(G / N)$ such that

$$
Y(\mu, S) Y^{*}=(1 \otimes M, 1 \otimes \nu) .
$$


For $s \in G$ and $f \in C_{0}(G / N)$, we compute

$$
\begin{aligned}
\left(1 \otimes \lambda_{s N}^{*}\right) Y U_{s} Y^{*}(1 \otimes M(f)) & =\left(1 \otimes \lambda_{s N}^{*}\right) Y U_{s} \mu(f) Y^{*} \\
& =\left(1 \otimes \lambda_{s N}^{*}\right) Y \mu\left(\mathrm{lt}_{s}(f)\right) U_{s} Y^{*} \\
& =\left(1 \otimes \lambda_{s N}^{*}\right)\left(1 \otimes M\left(\mathrm{lt}_{s}(f)\right)\right) Y U_{s} Y^{*} \\
& =(1 \otimes M(f))\left(1 \otimes \lambda_{s N}^{*}\right) Y U_{s} Y^{*}
\end{aligned}
$$

and a similar computation using $U_{s} S_{t N}=S_{t N} U_{s}$ shows that $\left(1 \otimes \lambda_{s N}^{*}\right) Y U_{s} Y^{*}$ also commutes with every $1 \otimes \nu_{t N}$. Thus $\left(1 \otimes \lambda_{s N}^{*}\right) Y U_{s} Y^{*}$ belongs to the commutant

$$
\left((1 \otimes(M \rtimes \nu))\left(C_{0}(G / N) \rtimes G / N\right)\right)^{\prime}=\left(1 \otimes \mathcal{K}\left(L^{2}(G / N)\right)\right)^{\prime}=B\left(\mathcal{H}_{2}\right) \otimes 1,
$$

and hence there is an operator $W_{s}$ on $\mathcal{H}_{2}$ such that $\left(1 \otimes \lambda_{s N}^{*}\right) Y U_{s} Y^{*}=W_{s} \otimes 1$, or equivalently such that $Y U_{s} Y^{*}=W_{s} \otimes \lambda_{s N}$. Straightforward calculations show that $W$ is a strongly continuous unitary representation of $G$. Since $Y \pi_{1}(a) Y^{\star}$ commutes with every $1 \otimes M(f)=Y \mu(f) Y^{*}$ and every $1 \otimes \nu_{t N}=Y S_{t N} Y^{*}$, there is also a representation $\pi_{2}$ of $A$ on $\mathcal{H}_{2}$ such that $Y \pi_{1}(a) Y^{*}=\pi_{2}(a) \otimes 1$. Now the calculation

$$
\begin{aligned}
Y\left(\pi_{1} \otimes \mu\right)(a \otimes f) Y^{*} & =Y \pi_{1}(a) \mu(f) Y^{*} \\
& =\left(\pi_{2}(a) \otimes 1\right)(1 \otimes M(f)) \\
& =\left(\pi_{2} \otimes M\right)(a \otimes f)
\end{aligned}
$$

gives the result.

REMARK 3. The argument in the last paragraph of the previous proof is the key step in proving that

$$
\left(A \otimes \mathcal{K}\left(L^{2}(G / N)\right), i_{A} \otimes M,\left(i_{G} \otimes \lambda\right) \rtimes(1 \otimes \nu)\right)
$$

is a crossed product for the system

$$
\left(A \otimes C_{0}(G / N), G \times(G / N),(\alpha \otimes \mathrm{lt}) \times(\mathrm{id} \otimes \mathrm{rt})\right) .
$$

From this, we deduce that the map

$$
\left(\left(i_{A} \otimes M\right) \rtimes\left(i_{G} \otimes \lambda\right)\right) \rtimes(1 \otimes \nu)
$$

is an isomorphism of $\left(\left(A \otimes C_{0}(G / N)\right) \rtimes_{\alpha \otimes l t} G\right) \rtimes_{\beta}(G / N)$ onto $\left(A \rtimes_{\alpha} G\right) \otimes \mathcal{K}\left(L^{2}(G / N)\right)$. Since the isomorphism

$$
\left(A \otimes C_{0}(G / N)\right) \rtimes_{\alpha \otimes \mid \mathrm{t}} G \cong\left(A \rtimes_{\alpha} G\right) \rtimes_{\widehat{\alpha} \mid}(G / N)
$$

of $\left[4\right.$, Theorem A.64] carries $\beta$ into the dual action $(\widehat{\alpha} \mid)^{\wedge}$ and (2.8) into the regular representation of $\left(\left(A \rtimes_{\alpha} G\right) \rtimes_{\widehat{\alpha} \mid}(G / N)\right) \rtimes_{(\hat{a} \mid)^{\wedge}}(G / N)$ into

$$
\left(A \rtimes_{\alpha} \mathcal{K}\left(L^{2}(G / N)\right)=\mathcal{K}\left(\left(A \rtimes_{\alpha} G\right) \otimes L^{2}(G / N)\right)\right.
$$


the injectivity of (2.8) says precisely that the restriction $\widehat{\alpha} \mid$ of the dual coaction is maximal in the sense of $[\mathbf{3}]$.

The maximality of $\widehat{\alpha} \mid$ can also be deduced from the general results in [10], but of course it is reassuring to have a direct argument. In a previous version of this paper, we deduced from [10, Proposition 7.1 and Corollary 7.2] that $\widehat{\alpha} \mid$ is maximal, and then proved Theorem 2 using Mansfield imprimitivity for maximal coactions [10, Theorem 5.3]. We are grateful to the referee of that version for suggesting the direct approach used here.

Theorems 1 and 2 give the following improvement to [9, Theorem 5]. (It is the absence of amenability hypotheses rather than the presence of $A$ which is the real improvement.)

COROLlary 4. Suppose that $\alpha$ is an action of a locally compact group $G$ on a $C^{*}$-algebra $A$ and $N$ is a closed normal subgroup of $G$. Let $(\pi, U)$ be covariant representation of $(A, N, \alpha)$ on $\mathcal{H}$. Then there exists a representation $V$ of $G$ on $\mathcal{H}$ such that $(\pi, V)$ is a covariant representation of $(A, G, \alpha)$ and $\left.V\right|_{N}=U$ if and only if there exists a representation $S$ of $G / N$ such that $(X-\operatorname{Ind}(\pi \rtimes U), S)$ is a covariant representation of $\left(\left(A \otimes C_{0}(G / N)\right) \rtimes_{\alpha \otimes \mathrm{lt}} G, G / N, \beta\right)$.

\section{REFERENCES}

[1] K. Deicke, D. Pask and I. Raeburn, 'Coverings of directed graphs and crossed products of $C^{*}$-algebras by coactions of homogeneous spaces', Internat. J. Math. 14 (2003), 773-789.

[2] S. Echterhoff, 'Duality of induction and restriction for abelian twisted covariant systems', Math. Proc. Camb. Phil. Soc. 116 (1994), 301-315.

[3] S. Echterhoff, S. Kaliszewski and J. Quigg, 'Maximal coactions', Internat. J. Math. 15 (2004), 47-61.

[4] S. Echterhoff, S. Kaliszewski, J. Quigg and I. Raeburn, 'A categorical approach to imprimitivity theorems for $C^{*}$-dynamical systems', Memoirs Amer. Math. Soc. 180 (2006), 1-169.

[5] S. Echterhoff, S. Kaliszewski and I. Raeburn, 'Crossed products by dual coactions of groups and homogeneous spaces', J. Operator Theory 39 (1998), 151-176.

[6] S. Echterhoff and J. Quigg, 'Full duality for coactions of discrete groups', Math. Scand. 90 (2002), 267-288.

[7] P. Green, 'The local structure of twisted covariance algebras', Acta Math. 140 (1978), 191-250.

[8] A. an Huef, S. Kaliszewski and I. Raeburn, 'Covariant representations of Hecke algebras and imprimitivity for crossed products by homogeneous spaces', (preprint) arXiv:math. 0A/0509291.

[9] A. an Huef and I. Raeburn, 'Twisted actions and the obstruction to extending unitary representations of subgroups', J. Pure Appl. Algebra 194 (2004), 299-309.

[10] S. Kaliszewski and J. Quigg, 'Mansfield imprimitivity for full crossed products', Trans. Amer. Math. Soc. 357 (2005), 2021-2042.

[11] S. Kaliszewski, J. Quigg and I. Raeburn, 'Duality of restriction and induction for $C^{*}$-coactions', Trans. Amer. Math. Soc. 349 (1997), 2085-2113. 
[12] K. Mansfield, 'Induced representations of crossed products by coactions', J. Funct. Anal. 97 (1991), 112-161.

[13] D. Pask, J. Quigg and I. Raeburn, 'Coverings of k-graphs', J. Algebra 289 (2005), 161-191.

[14] I. Raeburn and D.P. Williams, Morita Equivalence and Continuous-Trace $C^{*}$-Algebras, Math. Surveys and Monographs 60 (Amer. Math. Soc., Providence, R.I., 1998).

School of Mathematics and Statistics

The University of New South Wales

New South Wales 2052

Australia

e-mail: astrid@unsw.edu.au
Department of Mathematics and Statistics Arizona State University, AZ 85287-1804 United States of America e-mail: kaliszewski@asu.edu

School of Mathematical and Physical Sciences

University of Newcastle

NSW 2308

Australia

e-mail: iain.raeburn@newcastle.edu.au 